\title{
Patient Reported Experiences at a Swedish National Burn Centre
}

\author{
Laura Pompermaier, MD, PhD, * Emma Drake af Hagelsrum, MD, * Viktor Ydenius, MD, * \\ Folke Sjöberg, MD, PhD, ${ }^{*} \dagger$ Ingrid Steinvall, PhD, ${ }^{*}$ and Moustafa Elmasry, $\mathrm{MD}, \mathrm{PhD}$ *
}

\begin{abstract}
Previous studies have shown that burn patients were satisfied with the received care. Satisfaction was not strongly associated to burns or to psycho-social characteristics, suggesting that other factors, related to burn care specific aspects, may be important. The aim of this study was to analyze the independent effect of provided workload on the general satisfaction in adult patients at a Swedish national Burn Centre. The study population $(\mathrm{n}=122)$ included patients $\geq 18$ years, treated at the Linköping Burn Centre between 2016 and 2017. Experienced burn care was evaluated with the PS-RESKA survey (score range: 0-4), and provided workload was scored with the Burn Scoring System (BSC). Groups were compared with $\chi^{2}$ test, MW test, or Fisher's exact test. Multivariable logistic regression analyzed the independent effect of BSC on the outcome High Satisfaction (= score $\geq 3$ to the survey-question: “How would you score your global experience at the Burn Centre?"). In-patients (n = 60) had more often larger burns and required more workload than out-patients (median[IQR]: TBSA\% $=6.3$ [3-12.3] \% vs. $0.7[0.3-2] \%, p<.001 ; \mathrm{BSC}=65[25.5-135.5]$ vs. $6[4-9], p<.001)$. Both groups were highly satisfied with the experienced care (mean score $[S D]=3.68[0.57]$ vs. $3.41[0.77], p=.03$ ). Neither characteristics of the patients (age, sex), nor TBSA\% nor provided workload (BSC) were independently associated with High Satisfaction. Regardless of burn severity, demographics and provided workload, adult patients with burns were highly satisfied with the experienced burn care. This finding suggested that the reason of the satisfaction was multifactorial.
\end{abstract}

Research on burns management has predominantly focused on factors valuable to better the clinical outcomes after provided care, such as in-hospital mortality and duration of stay at the hospital, contributing to improve the standard of burn care worldwide. ${ }^{1-4}$ However, research on burn care has often been conducted from the care provider's perspective, overlooking patient reported experience measures (PREM). ${ }^{5}$

More than two decades ago, Norwegian researchers developed the Patient Satisfaction-Results and Quality (PS-RESKVA), a survey aiming to identify the major determinants of patient satisfaction after hospital care. ${ }^{6}$ The survey was then adapted for surgical patients with questions regarding four themes: global satisfaction with treatment (GS), perceived quality of contacts with the nursing staff (QCN), perceived quality of contacts with medical staff (QCM), and provision of adequate treatment information (INF). ${ }^{7}$ A decade

\footnotetext{
From the *Department of Hand Surgery, Plastic Surgery and Burns, Department of Biomedical and Clinical Sciences, Linköping University, Sweden; ${ }^{+}$Department of Anaesthesiology and Intensive Care, Linköping University, Sweden.

Conflict of interest statement. We have no conflicts of interest.

Address correspondence to Laura Pompermaier, MD, PhD, The Burn Centre Department of Hand and Plastic Surgery, Linköping University Hospital, 58185 Linköping, Sweden. Email: laura.pompermaier@liu.se

Funding: This study was done and funded by the Department of Hand Surgery, Plastic Surgery and Burns, and Linköping University, Linköping, Sweden.

(C) The Author(s) 2021. Published by Oxford University Press on behalf of the American Burn Association.

This is an Open Access article distributed under the terms of the Creative Commons Attribution-NonCommercial License (http://creativecommons.org/ licenses/by-nc/4.0/), which permits non-commercial re-use, distribution, and reproduction in any medium, provided the original work is properly cited. For commercial re-use, please contact journals.permissions@oup.com

doi:10.1093/jbcr/irab091
}

later, the PS-RESKVA was used in Uppsala (Sweden) to measure the experienced care among former burn patients, finding that satisfaction in relation to nursing staff was scored higher than other aspects of burn care. ${ }^{8}$ The same research group also showed that the psycho-social characteristics of the respondents contributed to predict their satisfaction, while burn severity did not. ${ }^{9}$ Furthermore, as the explained variance was low, the authors hypothesized that other factors related to the care itself affected patient satisfaction.

In the last ten years, treatment strategies and care organization has changed in Sweden, contributing to improve clinical outcomes after burn care. ${ }^{10-12}$

Since 2010 , the provision of specialized burn care in Sweden has been designated to two national Burn Centres, one in Linköping and one in Uppsala. The referral criteria are listed in the national guidelines and the catchment areas of the two centers are defined a priori. Patients are not consulted on the decision of being referred, nor of the center where to be referred.

At the Burn Centre in Linköping, the medical interventions provided to each patient are scored daily by the nursing staff using the Burn Scoring System (BSC): the workload delivered daily for each of the seven categories considered in the BSC (surveillance, ventilation, circulation, wound care, mobilization, clinical chemistry, infusion/injection) is scored from 0 to 4 points, while the operation time generates 2 points per hour. ${ }^{2}$ The BSC was originally developed to calculate the cost of the treatments, ${ }^{2,13}$ but has been also used to analyze effect of treatment on outcomes ${ }^{14}$ and possible inequity in the management of patients with burns. ${ }^{15}$

However, it remains to be investigated whether patients are satisfied with the care after the modified burn management, 
and whether their experience is associated with care related factors.

This study aimed therefore to analyze with the PS-RESKVA the reported experience of burn care received at the Linköping Burn Centre among former patients with burns, and to investigate independent association of general satisfaction with received interventions, assessed with the BSC.

\section{METHODS}

This cross-sectional study aimed to analyze the burn care experienced by patients at the Linköping Burn Centre using the standardized questionnaire PS-RESKVA. ${ }^{7,8}$ The original PS-RESKVA scores (on a rating scale from 0 to 4 ) contains 27 questions on four items: global satisfaction with treatment (GS), perceived quality of contact with the nursing staff (QCN), perceived quality of contact with the medical staff (QCM), and provision of adequate treatment information (INF). We included 17 of these questions in our analysis, because in our opinion, 10 of the 27 were redundant, or also required medical knowledge to obtain plausible answer. Furthermore, we added one more question that would allow us to score the whole burn care experience; "How would you score your global experience at the Burn Centre?." Demographic and clinical information were extracted from the local burn unit database and from medical records, and data on the received care were from the BSC. ${ }^{2,13}$

The survey in Swedish language was sent by post to the home address of all former patients managed at the Linköping Burn Centre between January 1, 2016 and December 31, 2017, except those without an available address, those transferred to another ward during the admission, and those who died before the discharge. The questionnaire was sent to in-patients after their discharge from the Burn Centre, and to out-patients at the end of the follow-up at the out-patient clinic. Those patients who were initially managed at the outclinic but were later admitted to the ward because of changed need of care, were classified as in-hospital patients. In-hospital patients discharged from the burn ward and who continued their follow up at the out-clinic, were classified as in-hospital patients.

The questionnaire was sent with a free of charge return envelope, and if no reply was received after two weeks, a reminder was sent. Patients who still did not reply after the reminder were considered as dropout. The study population consisted of patients $\geq 18$ years who completed the survey, while children were not included to avoid the bias caused by form completed by their guardians, but they were described separately as subgroup.

The variables coded as continuous were age (years), percentage of the total body surface area burned (TBSA\%), Burn Scoring System (BSC, score points), and duration of stay at the hospital (length of stay = LOS, days) for in-patients, and out-patient clinical visits (number) for out-patients.

The variables coded as categorical were gender (female/ male), presence of full thickness burns (FTB, yes/no), in-patient status (yes/no), cause of burns (electrical, explosion, contact, chemical, scalds, fire, other), and other causes, which included disorders that caused loss of skin (friction, degloving or skin disease). We considered the question: "How would you score your global experience at the Burn Centre?" as a proxy for the general satisfaction with the received care, and the outcome variable was High Satisfaction, dichotomized in yes by score $\geq 3$ (Highly satisfied patients), and in no by score $<3$ (Less satisfied patients).

\section{Statistics}

Descriptive data were presented as frequency (\%) or median (IQR) or mean (SD). Differences between demographic characteristics of responders and not-responders, male/female, in-/out-patients, and highly satisfied/ less satisfied patients were tested with Chi square test, Mann-Whitney test, t test, or Fisher's exact test, as appropriate. Data on how much the study population scored the questions of the survey (on a rating scale from 0 to 4 ) was reported as mean (SD). Differences on how the questions were scored among in- and out-patients, and among highly satisfied and less satisfied patients, were tested with t test.

Multivariable logistic regression was used to analyze in the study population the association between High Satisfaction and the following factors: age, sex, TBSA\% burned, presence of a full thickness burns (FTB), Burn Scoring System (BSC) and in-patient status. Multivariable logistic regression was also used to analyze among in-patients, factors that might be associated with High Satisfaction, such as age, sex, TBSA\%, presence of FTB, BSC and LOS; and among out-patients, the association of High Satisfaction with age, sex, TBSA\%, presence of FTB, BSC and number of out-clinic visits. In the subgroups of children, multivariable logistic regression was used to analyze the association between High Satisfaction and the following factors: age, sex, TBSA\% burned, Burn Scoring System (BSC) and in-patient status. The area under the Receiver Operating Characteristics (ROC) curve (AUC) was calculated for the four models.

Data were analyzed with STATISTICA 64 (version 13, Dell Inc., Round Rock, TX) and STATA (version 16.1, Stata Corporation LP College Station, TX). Probabilities of less than 0.05 were accepted as significant.

\section{RESULTS}

During the study period, a total of 534 patients with burns and skin loss caused by other conditions were treated at the Linköping Burn Centre. After exclusion of patients without an available address $(n=29)$, those who were transferred to another ward $(n=2)$, and those who died during the admission $(\mathrm{n}=13), 490$ surveys were sent out to former patients in every age (241 adults and 249 children). After exclusion of respondents younger than $18(\mathrm{n}=135)$, the study population consisted of the 122 adult patients who completed the survey, with a response rate of $51 \%(122 / 241)$. The study population was significantly older than the former patients who did not complete the questionnaire, with a median age (IQR) of 48.0 years (31.4-62.7) and 36.5 years (27.2-51.4), respectively, $(p<.001)$, while there were not differences in sex, TBSA\%, presence of FTB, type of care, and BSC (Supplementary Table S1). Most of the respondents were male $(\mathrm{n}=84,69 \%)$, and among sexes, age distribution was comparable (age: male $=48.0$ years [31.0-61.2], 
female $=49.1[37.2-70.6], p=.47)$, and there were no differences in burn size and depth (TBSA burned: male $=3 \%$ [0.5-6.7], female $=1.8 \%[0.6-4.8], p=.38$; presence of FTB: male $=19$, female $=5, p=.32)$. There was a significant difference in the causes of burns, with female patients having more frequently injuries caused by scalds $(\mathrm{n}=17,45 \%)$ and male by fire $(\mathrm{n}=30,36 \%), p=.009$. The care provided at the Burn Centre and measured with the Burn Scoring System (BSC) $)^{2}$ was comparable among sexes (BSC: male $=11[7-71]$, female $=14[5-56], p=.70$ ), and both sexes were mostly highly satisfied over the experienced burn care (High Satisfaction: male $=77[92 \%]$, female $=32[84 \%], p=.22)$. Median satisfaction was scored 4 points $(\mathrm{IQR}=3-4)$ on a rating scale from 0 to 4 , and no patient scored the global experience at the Burn Centre less than 2 points.

The characteristics of the study population and the comparison between in-patients and out-patients are shown in Table 1. Age and gender distribution were comparable among the groups $(p=.78$ and $p=.15$, respectively). Patients admitted for in-hospital care had a median LOS of 13 days (IQR: 6-23); they had larger and deeper burns and required more workload than out-patients $(p<.001)$. Scalds were the primary cause of injury among out-patients, fire among in-patients $(p=.002)$. The median number of visits at the out-patients clinic was of 3.5 (IQR: 2-5); only one out-patient had full thickness burns. Most of the patients were highly satisfied by the received care and there was no difference among in- and outpatients $(p=.08)$. Among the subgroup of patients younger than 18 , median age (IQR) was 1.8 years $(1.2-4.4), 61 \%$ of them were boys $(82 / 135)$, median TBSA\% was $1.9(0.5-4.4)$ and only 3 patients had presence of full thickness burns at the admission. Median BSC (IQR) was 10 (5-17) and most of patients (119/135) were highly satisfied with the experienced care (Supplementary Table S2).

The study population was highly satisfied with the experienced care [mean score (SD) to the question: "How would you score your global experience at the Burn Centre?" was 3.55 (0.68). When compared with out-patients, in-patients scored the entire care experience higher $(p=.03)$, whereas out-patients were more satisfied with the communication with the medical staff than in-patients $(p=.08)$. Supplementary Table S3 shows how patients scored the experienced care at the Linköping Burn Centre. Neither in the univariate nor in the multivariate analysis we were able to find any significant associations with high satisfaction scored by the entire study population (Table 2), nor in-patients or in out-patients. After adjustment for other factors, longer LOS and younger age showed a tendency $(p=.08$ and $p=.05)$ to be associated with high satisfaction among in-patients (Supplementary Table S4), while older age showed a tendency $(p=.08)$ among the out-patients (Supplementary Table S5). However, the in-patients model showed an excellent capacity to distingue between highly satisfied and less satisfied patients (AUC $=0.95$ [95\% CI: 0.85-1.00], in Supplementary Table S4), while the inclusion of out-patients reduced the models' discrimination (AUC $=0.76$ [95\% CI: 0.69-0.90] in Table 2 and $\mathrm{AUC}=0.73$ [95\% CI: 0.58-0.88] in Supplementary Table S5). Even in the subgroup of children, neither in the univariate nor in the multivariate analysis we were able to find any significant associations between age, sex, TBSA\%, BSC, in-patient status and high satisfaction (Supplementary Table S6).

Thirteen former patients ( $11 \%$ of the study population) were less satisfied with the global experience at the Burn

Table 1. Demographics characteristics of study population, comparing in-patients and out-patients

\begin{tabular}{|c|c|c|c|c|}
\hline & All Patients $(n=122)$ & Out-patients $(n=62)$ & In-patients $(n=60)$ & $P$ \\
\hline Male sex, $\mathrm{n}(\%)^{*}$ & $84(69 \%)$ & $39(63 \%)$ & $45(75 \%)$ & .15 \\
\hline Age, years** & $48.0(31.4-62.7)$ & $50.3(35.7-61.0)$ & $45.0(30.5-63.9)$ & .77 \\
\hline TBSA\%* * & $2.3(0.5-6)$ & $0.7(0.3-2)$ & $6.3(3-12.3)$ & $<.001$ \\
\hline Presence of FTB, $\mathrm{n}(\%)^{* * *}$ & $24(20 \%)$ & $1(2 \%)$ & $23(38 \%)$ & $<.001$ \\
\hline BSC, score points** & $11.5(6-65)$ & $6(4-9)$ & $65(25.5-135.5)$ & $<.001$ \\
\hline High satisfaction, $\mathrm{n}(\%)^{*}$ & $109(89 \%)$ & $52(84 \%)$ & $57(95 \%)$ & .08 \\
\hline
\end{tabular}

TBSA\%, percentage of total body surface area; FTB, full-thickness burns; BSC, Burn Scoring System.

High Satisfaction = patients who scored the question: "Were you satisfied with the care and treatment you received in the burn clinic?" 3 or more points (score range 0-4). Data are presented in number (\%) or median (IQR: 25-75 centiles).

${ }^{*}$ Chi square test; ${ }^{* *}$ Mann-Whitney U test; ${ }^{* * *}$ Fishers exact.

Table 2. Evaluation of factors associated with high satisfaction in the study population, $\mathrm{n}=122$

\begin{tabular}{|c|c|c|c|c|}
\hline & Unadjusted OR (95\% CI) & $P$ & Adjusted OR (95\% CI) & $P$ \\
\hline In-patient status & $3.65(0.95-14.01)$ & .06 & $0.69(0.09-5.16)$ & .72 \\
\hline Male sex & $2.06(0.64-6.62)$ & .22 & $1.90(0.56-6.52)$ & .31 \\
\hline Age & $1.01(0.98-1.04)$ & .61 & $1.00(0.97-1.04)$ & .84 \\
\hline TBSA\% & $1.40(0.99-1.97)$ & .06 & $1.35(0.88-2.06)$ & .17 \\
\hline Presence of FTB & $3.21(0.40-25.98)$ & .27 & $2.52(0.20-31.78)$ & .48 \\
\hline BSC & $1.02(0.99-1.05)$ & .14 & $1.01(0.97-1.04)$ & .66 \\
\hline
\end{tabular}

TBSA\%, percentage of total body surface area; BSC, Burn Scoring System.

High Satisfaction= patients who scored the question “How would you score your global experience at the Burn Centre?” 3 or more points (score range 0-4). Odds Ratio $(95 \%$ Confidence Interval $)=$ OR $(95 \%$ CI $)$. AUC $=0.76$ (95\% CI: 0.69-0.90). 
Centre and their mean score (SD) for the question, "How would you score your global experience at the Burn Centre?" was $2(0)$, when compared with $3.55(0.44)$ in the highly satisfied individuals $(p<.001)$. There were not differences among sex, age and presence of FTB among these groups, while the less satisfied patients had significantly smaller injuries than the highly satisfied (median TBSA\% [IQR] 0.5 [0.3-1.5] and $3.1[0.7-6.6], p<.001)$. Although not significant, most of the less satisfied patients were out-patients and required less workload, when compared with the highly satisfied patients (out-patients $=10 / 13$ and 54/109, $p=.08$; median [IQR] $\mathrm{BSC}=7[4-14]$ and $12[6-75], p=.06)$, as shown in Table 3. Supplementary Table S7 compare the answers to the PS-RESKVA questions between highly satisfied and less satisfied individuals. Despite even less satisfied patients scored more than 3 questions regarding the quality of the contact with the nursing staff, their scores in this item were significantly lower than those in the highly satisfied group. Furthermore, communication was a problem with both the nursing and the medical staff, and they experienced that doctors and nurses cared less, as the highly satisfied patients felt.

Supplementary Table S8 shows the association between the score values of "How would you score your global experience at the Burn Centre?" and those of each of the other questions calculated on the entire study population. All but three questions ("Did you get instructions about what to do yourself to improve or to prevent aggravation?," $\mathrm{r}=.13$, "Did one physician have the responsibility for you?," $\mathrm{r}=.12$, "Were you prepared about difficulties in the home situation after hospital treatment?," $\mathrm{r}=.09$ ) showed a significant association. The strongest association was found with the two questions: "Were you satisfied with the treatment?" $(\mathrm{r}=.42$, coefficient $=0.56, p<.001)$ and "Did the medical staff convey a caring attitude?" $(\mathrm{r}=.42$, coefficient $=.45, p<.001)$.

\section{DISCUSSION}

The International Society for Burn Injuries (ISBI) strongly recommended that severe and/or pediatric burns be managed by an organized system, when the resources allow it, ${ }^{16}$ and indeed admission to dedicated burn units has been associated with better survival. ${ }^{17}$ In Sweden, patients with burns who require specialized burn care are referred to one of the two national designed burn centers, based on their catchment area.
The improved outcomes of the last decades at the Linköping Burn Centre, as well as in other Burn Centres of high-income countries, ${ }^{14,18-20}$ have confirmed the effectiveness of centralization of burns care to facilities where specialized knowledge, infrastructures, staff and research are concentrated. Implementation strategies adopted in Linköping, such as early excision, presence of dedicated medical and nursing staff, management of smaller injuries at the out-patients clinic, and short-stay admissions, are comparable to those made in other international burn centers, which supports generalizability of our findings. ${ }^{21-23}$ However, if no one doubts the benefits of dedicated burn units, we found that just few care givers asked patients how their experience at the specialized burn center was and if they were satisfied with it. ${ }^{8,24,25}$ This question is not mere courtesy but is necessary to identify factors related to the provided care that need to be improved, and indeed patient trust and good communication with care givers have been associated with better compliance with the therapies and better outcomes. ${ }^{26}$

Also burn management might be favored by a positive attitude of patients towards burn care, because it requires a more or less long period of surgical procedures, changes of dressing, tricky analgesic and antibiotic therapies, and rehabilitation. For these reasons, the evaluation of patient satisfaction with the care received should be systematically included in the assessment of the quality of burn care. Burn societies, such as the American Burn Association (ABA) or the European Burn Association (EBA), offer programs to verify the quality of care delivered by burn centers, from the management of the acute injury throughout the rehabilitation process. The verification indicates to patients and their families that the center provides high quality patient care. However, these criteria that are evidence-based and established by expert consensus, do not include standards for assessing how patients themselves perceived the quality of burn care. To get a comprehensive evaluation of the quality of burn care, we think necessary to consider the patients' perspective, and we suggest including a requirement to use a validated questionnaire, such as the PS-RESKVA, among burn societies verification criteria. In addition, as medical services are a part of the market and nowadays, in the era of social media and open communication, the need to get feedback from customers is increasing, information about patients satisfaction plays an important role in evaluating the quality of care in a given facility. Therefore, future implementations in burn care should be developed

Table 3. Comparison between highly satisfied patients and less satisfied

\begin{tabular}{|c|c|c|c|}
\hline & Highly Satisfied Patients $(\mathrm{n}=109)$ & Less Satisfied Patients $(\mathrm{n}=13)$ & $P$ \\
\hline Male sex, $\mathrm{n}(\%)^{*}$ & $77(71 \%)$ & $7(54 \%)$ & .22 \\
\hline Age, years** & $48.3(31.4-63.6)$ & $44.3(27.6-52.9)$ & .48 \\
\hline TBSA $\% * *$ & $3.1(0.7-6.6)$ & $0.5(0.3-1.5)$ & $<.001$ \\
\hline Presence of FTB, $\mathrm{n}(\%)^{* * *}$ & $23(21 \%)$ & $1(0.1 \%)$ & .50 \\
\hline Out-patients, $\mathrm{n}(\%)^{* * *}$ & $52(48 \%)$ & $10(77 \%)$ & .08 \\
\hline BSC, score points** & $12(6-75)$ & $7(4-14)$ & .06 \\
\hline
\end{tabular}

TBSA, total body surface area; FTB, full-thickness burns; BSC, Burn Scoring System.

*Chi square test, ${ }^{* *}$ Mann-Whitney U test, *** Fishers exact.

Data are presented in number (\%) or median (IQR: 25-75 centiles). Highly satisfied patients= those who scored the question "How would you score your global experience at the Burn Centre?" $\geq 3$ (score range 0-4). Less satisfied patients= those who scored the question "How would you score your global experience at the Burn Centre?" $<3$. 
based also on the opinion of patients / customers, which leads to an increased need of evaluating versatility and effectiveness of tools that record patients 'feedback in burn centers, worldwide. Previous Swedish studies have shown that few patients reported negative emotional experiences during burn care, although a sense of powerlessness was described by severely injured. ${ }^{24}$ Furthermore, the sense of insecurity affected negatively the interaction with the care providers, while the feeling to cause nuisance influenced the contact with the nursing staff. Former patients appreciated more the relationship to the nursing staff, when compared with the quality of the interaction with the medical staff, or of the received information, or of the whole experience at the burn unit. ${ }^{8}$ Surprisingly, neither burns characteristics nor psycho-social factors were strongly correlated with satisfaction, suggesting that other factors, probably linked to the care itself, influence the experiences quality of received care. 9

With this study therefore, beside to analyze the quality of care experienced by patients at the Linköping Burn Centre, we aimed to investigate whether the provided workload affected their satisfaction. To the best of our knowledge, this is the first study to investigate whether medical interventions provided during burn care affected the patient reported satisfaction. Workload was assessed with the Burn Scoring System (BSC), a validated method to score interventions provided to patient with burns. The BSC is not context-specific, but fits burn services, independently of their location, size, and available resources. We suspected that in-patients with burns that required more procedures and longer hospitalization, scored the entire experience lower than out-patients with less severe injuries, but we were wrong. Not only were most of patients in both groups highly satisfied with the received care, but indeed in-patients scored the global experience slightly higher than out-patients. This much positive conclusion reflects what was observed in a Spanish burn unit, where in-patients scored high the care received, in particular with regards of subjective qualities of the personnel, such as courtesy, ability to inspire trust and interest in patients. ${ }^{25}$ However, in our study outpatients seemed to be more satisfied with the communication with the medical staff and better informed on management of late complications than in-patients. A probable explanation of this finding is that out-patient visits are scheduled by appointment only, which allows to dedicate them enough time to interact and communicate. We found eventually that neither patients characteristics (age, sex, admission status), nor burn factors (TBSA\% burned, presence of FTB), nor provided workload (BSC) were independently associated with high satisfaction. Thirteen patients scored the question "How would you score your global experience at the Burn Centre?" less than 3 and were therefore categorized as less satisfied. There were not demographic differences between them and the more satisfied individuals, apart from the fact that these last had larger burns and the tendency (although not significant) to be in-patients and caused more workload. The items scored lower by the less satisfied group were mostly related to the quality of the contact with the nursing staff, which confirms the importance for the patients of the relationship with nurses, and the communication with the entire staff at the Burn Centre. Also, the less satisfied patients felt that the staff had a less caring attitude, as compared with the highly satisfied group. These unexpected findings will be useful to further improve the quality of care provided at our center. We will certainly focus on improving communication with patients even more, whereas it is more complex to work on changing the perception on the caring attitude of our staff, as this insight is based on a very personal opinion, and we would need more specific and contextualized information to develop an implementation strategy.

In this regard, we find particularly effective the Kano model, a qualitative method adopted by the Spanish colleagues. ${ }^{27}$ They developed a questionnaire focused on the experienced care at the burn unit, based on the findings of in-depth interviews with patients and their relatives. The survey was then distributed to the group of patients who participated in the interview and to other former burn patients. The answers were context-specific and useful to identify area of improvement and to develop implementation strategies ad-hoc. ${ }^{25}$ In sum, patients at the Linköping Burn Centre seem to be highly satisfied with the experienced care and the cause is most likely multifactorial. Further research is necessary to identify strengths and weaknesses in the management of burn care, suggesting context-specific solutions.

\section{Limitations}

This study has several limitations. First, only half of patients answered the survey and therefore the findings are not generalizable to all patients admitted at the Linköping Burn Centre. However, apart from the difference of age, with respondent being significantly older than non-responders, there were not differences in sex, burn severity (TBSA\% and presence of FTB), type of care (in- or out-patient care), and provided workload (BSC) among the two groups. For these reasons we retain the findings representative for the whole adult population admitted at our Centre during the study period.

Second, our study cohort does not include children and this choice was made to avoid a clear bias; in case of children, the questionnaire is mostly completed by their care givers who give their own version of their children's experience.

In fact, a recent Swedish study has shown that parent's rating of the quality of burn care received by their children was negatively affected by parent's fear, symptoms of posttraumatic stress, and depression. ${ }^{28}$ In our study, the median age of the subgroup of children was 1.8 years $(\mathrm{IQR}=1.2-4.4)$ and therefore, it is reasonable to assume that the parents not only influenced the responses, but were probably also the actual respondents. With this study, we aimed to investigate the patients' own experiences of care.

Finally, the most obvious limitation consists in the survey used for this study. We chose the PS-RESKVA form because it is validated for the use in burn care and had previously been utilized in a Swedish context. However, some of the 27 questions included in its original version explore the same topic repeatedly without adding new information, and some other require medical knowledge to be answered in a plausible way. For our analysis, we selected 17 of the 27 questions limiting the redundancy of the treated topic, and we have added one question ("How would you score your global experience at the Burn Centre?") that could evaluate the whole experience. We found that only 3 of the 17 questions were not significantly associated with that, which was presumed to summarize 
the whole experience (Supplementary Table S3). Although we have already reduced the number of the questions, we think that these could be further diminished by combining those formulated in a slightly different form, but not in content. Furthermore, reducing the number of questions could facilitate participation in the questionnaire, and could contribute increase the response rate.

\section{CONCLUSION}

Adults with burns are highly satisfied with the experienced care at a national burn center in Sweden, whether they are in-patients or out-patients. Neither patient characteristics, not burn related factors, nor the amount of provided interventions were independent factors for high satisfaction with the global experience at the Burn Centre. These findings suggest that the cause of high satisfaction with the experienced care was multifactorial.

\section{SUPPLEMENTARY DATA}

Supplementary data is available at Journal of Burn Care \& Research online.

\section{REFERENCES}

1. Ahuja RB. ISBI Practice guidelines for burn care: editorial. Burns 2016;42:951-2.

2. Sjöberg F, Danielsson P, Andersson L et al. Utility of an intervention scoring system in documenting effects of changes in burn treatment. Burns 2000;26:553-9.

3. Steinvall I, Elmasry M, Fredrikson M, Sjoberg F. Standardised mortality ratio based on the sum of age and percentage total body surface area burned is an adequate quality indicator in burn care: an exploratory review. Burns 2016;42:28-40.

4. Chong SJ, Kok YO, Choke A, Tan EWX, Tan KC, Tan BK. Comparison of four measures in reducing length of stay in burns: an Asian centre's evolved multimodal burns protocol. Burns 2017;43:1348-55.

5. Hodgkinson EL, Boullin P, Heary S, Grant B. Development and pilot of a burns-specific patient-reported experience measure. Burns 2019;45:1600-4.

6. Guldvog B, Hofoss D, Pettersen KI, Ebbesen J, Rønning OM. [PS-RESKVA (Patient Satisfaction, Results and Quality)-patient satisfaction in hospitals]. Tidsskr Nor Laegeforen 1998;118:386-91.

7. Sørlie T, Sexton HC, Busund R, Sørlie D. Predictors of satisfaction with surgical treatment. Int J Qual Health Care 2000;12:31-40.
8. Wikehult B, Kildal M, Willebrand M. Patient satisfaction with burn care 1-6 years after injury. Burns 2008;34:783-90.

9. Wikehult B, Ekselius L, Gerdin B, Willebrand M. Prediction of patient satisfaction with care one year after burn. Burns 2009;35:194-200.

10. Elmasry M, Steinvall I, Thorfinn J et al. Scald management protocolsoutcome differences in two different time periods using different treatment strategies. Ann Burns Fire Disasters 2016;29:139-43.

11. Elmasry M, Steinvall I, Abdelrahman I, Olofsson P, Sjoberg F. Changes in patterns of treatment of burned children at the Linkoping Burn Centre, Sweden, 2009-2014. Burns 2017;43:1111-9.

12. Svee A, Jonsson A, Sjöberg F, Huss F. Burns in Sweden: temporal trends from 1987 to 2010. Ann Burns Fire Disasters 2016;29:85-9.

13. Abdelrahman I, Elmasry $M$, Fredrikson $M$, Steinvall I. Validation of the burn intervention score in a National Burn Centre. Burns 2018;44:1159-66.

14. Abdelrahman I, Elmasry M, Steinvall I, Fredrikson M, Sjoberg F. Improvement in mortality at a National Burn Centre since 2000: was it the result of increased resources? Medicine (Baltim) 2017;96:e6727.

15. Pompermaier L, Elmasry M, Abdelrahman I, Fredrikson M, Sjöberg F, Steinvall I. Are there any differences in the provided burn care between men and women? A retrospective study. Burns Trauma 2018;6:22.

16. Committee IPG, Steering S, Advisory S, ISBI Practice Guidelines for Burn Care. Burns 2016;42:953-1021.

17. Win TS, Nizamoglu M, Maharaj R, Smailes S, El-Muttardi N Dziewulski P. Relationship between multidisciplinary critical care and burn patients survival: a propensity-matched national cohort analysis. Burns 2018;44:57-64.

18. Pompermaier L, Steinvall I, Fredrikson M, Sjöberg F. Inclusion of coexisting morbidity in a TBSA $\%$ and age based model for the prediction of mortality after burns does not increase its predictive power. Burns 2015;41:1868-76.

19. Smolle C, Cambiaso-Daniel J, Forbes AA et al. Recent trends in burn epidemiology worldwide: a systematic review. Burns 2017;43:249-57.

20. Zavlin D, Chegireddy V, Boukovalas S et al. Multi-institutional analysis of independent predictors for burn mortality in the United States. Burns Trauma 2018;6:24.

21. Elmasry M, Steinvall I, Thorfinn J et al. Temporary coverage of burns with a xenograft and sequential excision, compared with total early excision and autograft. Ann Burns Fire Disasters 2016;29:196-201.

22. Elmasry M, Steinvall I, Thorfinn J, Abdelrahman I, Olofsson P, Sjoberg F. Staged excisions of moderate-sized burns compared with total excision with immediate autograft: an evaluation of two strategies. Int J Burns Trauma 2017;7:6-11.

23. Engrav LH, Heimbach DM, Rivara FP et al. Harborview burns-1974 to 2009. PLoS One 2012;7:e40086.

24. Wikehult B, Hedlund $M$, Marsenic $M$, Nyman S, Willebrand $M$. Evaluation of negative emotional care experiences in burn care. J Clin Nurs 2008;17:1923-9.

25. Gómez Martín C, Garcia Morato RA, de Los Reyes Cortes J, FernandezCanamaque JL, Holgun P. Patient satisfaction in a Spanish burn unit. Burns 2019. 45:341-7.

26. Ward P. Trust and communication in a doctor-patient relationship: a literature review. Arch Med 2018;3:36.

27. Materla T, Cudney EA, Antony J. The application of Kano model in the healthcare industry: a systematic literature review. Total Qual Manag Bus Excel 2019;30:660-81.

28. Willebrand M, Sjöberg F, Huss F, Sveen J. Parents' perceived quality of pediatric burn care. J Crit Care 2018;43:256-9. 